\title{
Completion gastrectomy with esophagojejunostomy for management of complications of benign foregut surgery
}

\begin{abstract}
Background: With the worldwide epidemic of obesity, an increasing number of bariatric operations and antireflux fundoplications are being performed. Despite low morbidity of the primary foregut surgery, completion gastrectomy may be necessary as a definitive procedure for complications of prior foregut surgery; however, the literature evaluating outcomes after completion gastrectomy with esophagojejunostomy (EJ) for benign diseases is limited. We present our experience of completion gastrectomy with Roux-en-Y EJ in the setting of benign disease at a single tertiary center. Methods and Procedures: All patients who underwent total, proximal, or completion gastrectomy with EJ for complications of benign foregut surgery from January 2006 to December 2015 were retrospectively identified. All cancer operations were excluded. Results: There were 23 patients who underwent gastrectomy with EJ (13 laparoscopic EJ [LEJ] and 10 open EJ). The index operations included 12 antireflux, 9 bariatric, and 2 peptic ulcer disease surgeries. Seventy-eight percent of patients had surgical or endoscopic interventions before EJ, with a median of one prior intervention and a median interval from the index operation to EJ of 25 months (interquartile range 9-87). The 30-day perioperative complication rate was $30 \%$ with $17 \%$ classified being major (Clavien-Dindo $\geq$ III) and no 30-day perioperative mortality. Comparing laparoscopic and open approaches showed similar operative times, estimated blood loss, and overall complication rate. LEJ was associated with a shorter length of stay (LOS) $(\mathrm{P}<.001)$, fewer postoperative ICU days $(\mathrm{P}=.002)$, fewer 6-month complication rates $(\mathrm{P}<.007)$, and decreased readmission rate $(\mathrm{P}=.024)$. Conclusion: Our series demonstrates that $\mathrm{EJ}$ is a reasonable option for reoperative foregut surgery. The laparoscopic approach appears to be associated with decreased LOS and readmissions.
\end{abstract}

Keyword: Roux-en-Y; Completion gastrectomy; Complications; Esophagojejunostomy; Foregut surgery 